\title{
Adverse Event Toxicity Grade
}

National Cancer Institute

\section{Source}

National Cancer Institute. Adverse Event Toxicity Grade. NCI Thesaurus. Code C78605.

The toxicity grade value of the adverse event. 